\section{Peptoid special delivery}

\section{By Michael J. Haas, Senior Writer}

Researchers at the University of Karlsruhe have designed two peptoids that differentially deliver payloads to intracellular destinations in vitro without any toxic effects. The technique could produce carriers that deliver drugs exclusively to their intracellular targets-if peptoids prove to be as promising in vivo as they are in vitro.

Intracellular targets pose two challenges for therapeutic developers: getting compounds to penetrate the cell membrane and, once inside, having them actually find the target. Small molecules must typically exploit surface receptors to enter cells, but certain types of peptides can enter cells without receptor help. Over the past two decades, several classes of peptides and peptide-like molecules have been developed as carriers to deliver drug payloads to intracellular targets. But such carriers must often straddle a fine line between in vivo stability and toxicity.

Because they are structurally similar to natural peptides, synthetic peptides tend to be nontoxic. However, they can be readily metabolized in the blood, or degraded in cells-just like natural peptides-before delivering their payloads.

Peptidomimetics are more metabolically stable because of their non-natural structures, but for that reason they are also more likely to accumulate to toxic levels in cells or organs.

Peptoids, a class of peptidomimetics, have occasionally attracted interest as therapeutic agents, and a few studies have examined their cell-penetrating capabilities and suggested that they could function as drug carriers. ${ }^{1,2}$ But according to Stefan Braese, leader of the Karlsruhe research team and professor of organic chemistry at the university and the Karlsruhe Institute of Technology, peptoids have not been widely studied, in part because they are not as easily synthesized as other peptidomimetics or peptides.

"Peptoids should be more stable [with respect] to intracellular degradation than peptides," Braese told SciBX. "But peptoid construction needs at least some chemical expertise and most biologists might not be able or willing to synthesize these compounds. The building blocks have to be synthesized; they are not commercially available like amino acids."

In the Jan. 24 online edition of The Journal of Medicinal Chemistry, Braese and his team described the design and in vitro assessment of two peptoids that they say are metabolically stable and nontoxic and can preferentially deliver drug payloads to the cell cytoplasm or nucleus. ${ }^{3}$ This method could be used to fine tune the cellular uptake and intracellular destinations of these and other peptoid carriers.
The building block of any peptoid is an amino acid that has a side chain bound to the amide nitrogen - a feature that distinguishes it from a natural amino acid. Peptoids consist of monomeric sequences of these building blocks.

"We think that in the near future these building blocks will be available, like amino acids used for peptide synthesis," Braese said. "In addition, newly discovered methods such as microwave activation used by other groups will allow faster access to peptoids."

In designing the peptoids, Braese's team took into account three structural features relating to the cell-penetrating effectiveness of a peptide or peptide-like molecule: the importance of both a high content of positively charged side chains and a particular spatial arrangement of those side chains; and the relative unimportance of the amino acid backbone used.

The team built its peptoids with a backbone of five or six residues of glycine-the simplest amino acid. One peptoid had amino side chains; the other, guanidinium side chains. Both types of side chains are positively charged.

Next, the team linked each peptoid carrier to a fluorophore payload and used fluorescence-activated cell sorting, a type of flow cytometry, to measure the in vitro uptake in two human cell lines. Two established assays assessed cytotoxicity for each peptoid-fluorophore conjugate and each peptoid alone.

The results demonstrated only minor toxicity levels, which researchers attributed to the fluorophore payload rather than the peptoids. More significantly, researchers found that the two peptoids differed in their rates of uptake and where they accumulated in the cell-the amino peptoid localized in the cytoplasm and the guanidinium peptoid in the nucleus.

Thus, the authors reported that "the uptake rate and destination of the carriers can be tuned by just modifying the side chain functionality," making peptoids attractive carriers for intracellular delivery.

The use of designed peptoids as drug carriers should be extendable to any therapeutic area. For example, Braese's team has assessed how well its peptoids can deliver a variety of cancer drugs in vitro and in vivo. But peptoid carriers could be especially attractive to antisense companies taking aim at intranuclear molecules, which are notoriously difficult targets.

Antisense companies contacted by SciBX thought the peptoid technology held potential for commercial drug development, provided that further studies addressed two key issues: the mechanism of uptake and the in vivo toxicity.

"The paper represents a thoughtful and careful evaluation" of amino peptoids versus guanidinium peptoids, said Patrick Iversen, SVP of R\&D at antisense company AVI BioPharma Inc. "The strength of the work is the elegant chemistry and the well-controlled nature of the studies."

Iversen said he would feel more confident about the commercial potential of the peptoids if he knew more about the mechanisms of peptoid uptake and distribution, neither of which were addressed in the $J M C$ paper. "Investigations into how a delivery molecule may distribute to various subcellular compartments or to different cell types is the topic of greatest current interest," he said. 
Iversen said the factors affecting uptake could vary considerably between cell types, organs and even species, adding that understanding the mechanism of cell uptake could help improve the design of animal studies.

Tina Schroeder, a member of the Karlsruhe research team, told SciBX that the group's follow-on uptake studies have shown that one uptake mechanism of the amino peptoid is endocytosis: the peptoid interacts with the cell membrane and is pulled into the cell within an endosome-a self-contained compartment that renders the carrier and payload unavailable to the rest of the cell until the endosome breaks down.

"Endosomal trapping has pros and cons," Braese said. "On the pro side, it might store the compound for longer release. On the con side, the compound might not reach its target."

Schroeder said additional uptake studies on both peptoids, via selective blocking of possible pathways and staining of different cell organelles, will determine whether other uptake mechanisms play a role.

AVI's Iversen also said that the toxicity of peptoids is best determined in animals. "We and others have observed renal toxicity as the most sensitive measure of toxicity" of guanidinium-rich peptides used to deliver antisense oligomers, he said.

"This is not really a problem in the paper," he said, "but more in how extensively the authors' observations may be interpreted."

AVI's technology uses cell-penetrating peptides to deliver phosphorodiamidate morpholino oligo (PMO) payloads to cells. PMOs are similar in function to conventional antisense oligonucleotides but have chemical structures that make them less susceptible to metabolism and intracellular degradation.

AVI's AVI-4658 NeuGene AO is in a Phase I/II trial for Duchenne muscular dystrophy. The company has not disclosed a mechanism of action. AVI-5126, a NeuGene antisense compound targeting c-myc mRNA, is in a Phase II trial for ex vivo treatment of restenosis and saphenous veins in coronary artery bypass graft surgery, with completion expected by YE08. AVI expects to start a Phase I/II trial of the same compound for restenosis following angioplasty early this year.

Frank Bennett, SVP of research at antisense company Isis Pharmaceuticals Inc., agreed that thorough in vivo studies would be needed before concluding that the peptoids were nontoxic.

"This paper addressed one level of toxicity-that is, in cells," Bennett said. "The next level is toxicity studies in animals. Mechanisms of toxicity can be quite different in animals versus cells, because there are different, enhancement," he said.

more complex interactions, such as with blood proteins, and even different sensitivities between species," that cells cannot model.

Bennett said that, along with toxicity, animal studies could provide two other components that are crucial to a full assessment of carriers: pharmacology and pharmacokinetics.

"You need all three to write the equation showing that this technology brings value

Isis has multiple antisense compounds in development for a range of indications.

In January, Isis granted an exclusive, worldwide license to Genzyme Corp. to develop and commercialize mipomersen, a second-generation antisense compound targeting apolipoprotein B-100 (Apo B-100) for familial hypercholesterolemia. The companies expect to begin a Phase III trial this year.

The newest addition to the Isis pipeline, ISIS 388626, is a sodium-glucose cotransporter-2 inhibitor in preclinical development for diabetes.

Since submitting the JMC paper, Braese said his team has performed in vivo mouse studies on the peptoid-fluorophore conjugates and observed no short-term toxic effects. The team has also obtained what the researchers said are encouraging results from some preliminary pharmacological and pharmacokinetic studies in mice.

Next they are planning longer-term toxicological studies that could determine whether peptoids cause damaging reactive oxygen species to build up within cells.

The results for in vitro and in vivo delivery of anticancer drugs by peptoid carriers will be disclosed in Schroeder's upcoming dissertation.

Braese said the university has no immediate plans to file patents on the peptoid technology.

\section{REFERENCES}

1. Wender, P. et al. Proc. Natl. Acad. Sci. USA 97, 13003-13008 (2000)

2. Peretto, I. et al. Chem. Commun. 18, 2312-2313 (2003)

3. Schroeder, T. et al. J. Med. Chem.; published online Jan. 24, 2008; doi:10.1021/jm070603m

Contact: Stefan Braese, Institute of Organic Chemistry, University of Karlsruhe, Karlsruhe, Germany

e-mail: braese@ioc.uka.de

COMPANIES AND RESEARCH INSTITUTIONS MENTIONED

AVI BioPharma Inc. (NASDAQ:AVII), Portland, Ore.

Genzyme Corp. (NASDAQ:GENZ), Cambridge, Mass.

Isis Pharmaceuticals Inc. (NASDAQ:ISIS), Carlsbad, Calif.

Karlsruhe Institute of Technology, Karlsruhe, Germany

University of Karlsruhe, Karlsruhe, Germany 